\title{
THE INTERNAL WAVE DRIVEN DYNAMO IN ACCRETION
}

\section{DISKS}

\author{
E.T. VISHNIAC \\ The University of Texas, Austin, TX 78712 USA
}

\begin{abstract}
We present the results of recent work on a model of angular momentum transport in thin, ionized, accretion disks. In particular, we consider three physical effects, each of which can be represented in terms of a local MHD mode in such a disk. First, we discuss the generation and propagation of internal modes within accretion disks, pointing out certain features which make them particularly promising as the driving force behind a strong, fast dynamo in accretion disks. Second, we point ou that the magnetic shearing instability (MSI) first discussed by Velikhov, and more recently by Balbus and Hawley in the specific context of these disks, provides a natural saturation mechanism for any disk dynamo, leading to an approximate equality between the dimensionless viscosity and the ratio of the dynamo growth rate to the local shear. Third, we argue that magnetic buoyancy is largely suppressed by the turbulence generated by the shearing instability. This prevents it from removing magnetic flux from the disk any faster than random turbulent diffusion. We find that the dimensionless viscosity $\alpha$ scales as $(H / r)^{4 / 3}$, where $H$ is the disk height and $r$ is its radius.
\end{abstract}

\section{Introduction}

One of the more puzzling problems in astrophysics is the question of how angular momentum is transported outward in accretion disks. Such disks are believed to be ubiquitous in the universe and to account for a wide variety of energetic phenomena, but the basic physics underlying the radial movement of matter remain poorly understood. Without an understanding of this process we are unable to understand the production of radiant energy in accretion disks or their internal dynamics. Here we will report on recent work aimed at producing a working model of angular momentum transport in these systems (Vishniac and Diamond 1989; Vishniac, Jin and Diamond 1990; Vishniac and Diamond 1992).

We start with a statement of the problem that we wish to solve. First, we are concerned with thin accretion disks, i.e. those for which the disk thickness $H$ is much less than the radius $r$. This is not because we consider thick disks to be rare, but because we use $(H / r)$ as an ordering parameter. Our model should not be extended to disks where this is of order unity. Second, we assume that the local rotational frequency $\Omega$ is essentially Keplerian, i.e. $\Omega^{2}=G M_{*} / r^{3}$. If the disk is thin than this is equivalent to assuming that $M_{d i s k} \ll M_{*}$ Third, we will assume that our disks have negligible self-gravity. In other words, their vertical gravity is approximately $-z \Omega^{2}$, where $z$ is the distance to the midplane and $\Omega$ is the local rotational frequency. This implies that $M_{\text {disk }} \ll(H / r) M_{*}$. A disk that violates this condition will suffer from local gravitational instabilities which are outside the scope of this paper. As a consequence of these conditions we have $c_{s} \sim H \Omega$ where $c_{s}$ is the sound speed at the disk midplane. Fourth, we ignore any externally imposed magnetic field. Fifth, we assume that the disk is perfectly conducting. These conditions need not be met everywhere within a disk. Rather we expect that our treatment of the problem will be valid for those regions within a disk where these conditions are met. Viewed in this light these are not particularly onerous conditions and will apply to the majority of visible systems. 
The traditional treatment of accretion disks relies on the introduction of a phenomenological viscosity given by $\nu=\alpha c_{s} H$ (Shakura and Sunyaev 1973). We do not rely on this approach, but for convenience we will quote our results in terms of an equivalent dimensionless viscosity $\alpha$.

Our model is built up from a number of discrete elements. First, we argue that the physical conditions in accretion disks favor the presence of low $m$ internal waves whose amplitude is determined by nonlinear dissipation. Second, we point out that such waves are capable of driving an $\alpha-\Omega$ dynamo leading to the appearance of a large scale, mostly azimuthal, magnetic field. Third, we point out that shearing instabilities in this field will cause the saturation of the dynamo, as well as the outward transport of angular momentum. Fourth, we note magnetic buoyancy, frequently cited as an important limitation on the growth of magnetic fields in disks, is greatly moderated by the shearing instability so that it is prevented from playing an important role in the field evolution. Finally we present a series of scaling arguments for the internal properties of accretion disks based on this model. The flowchart below illustrates the way the various elements of this model interact to drive mass inward in an accretion disk.

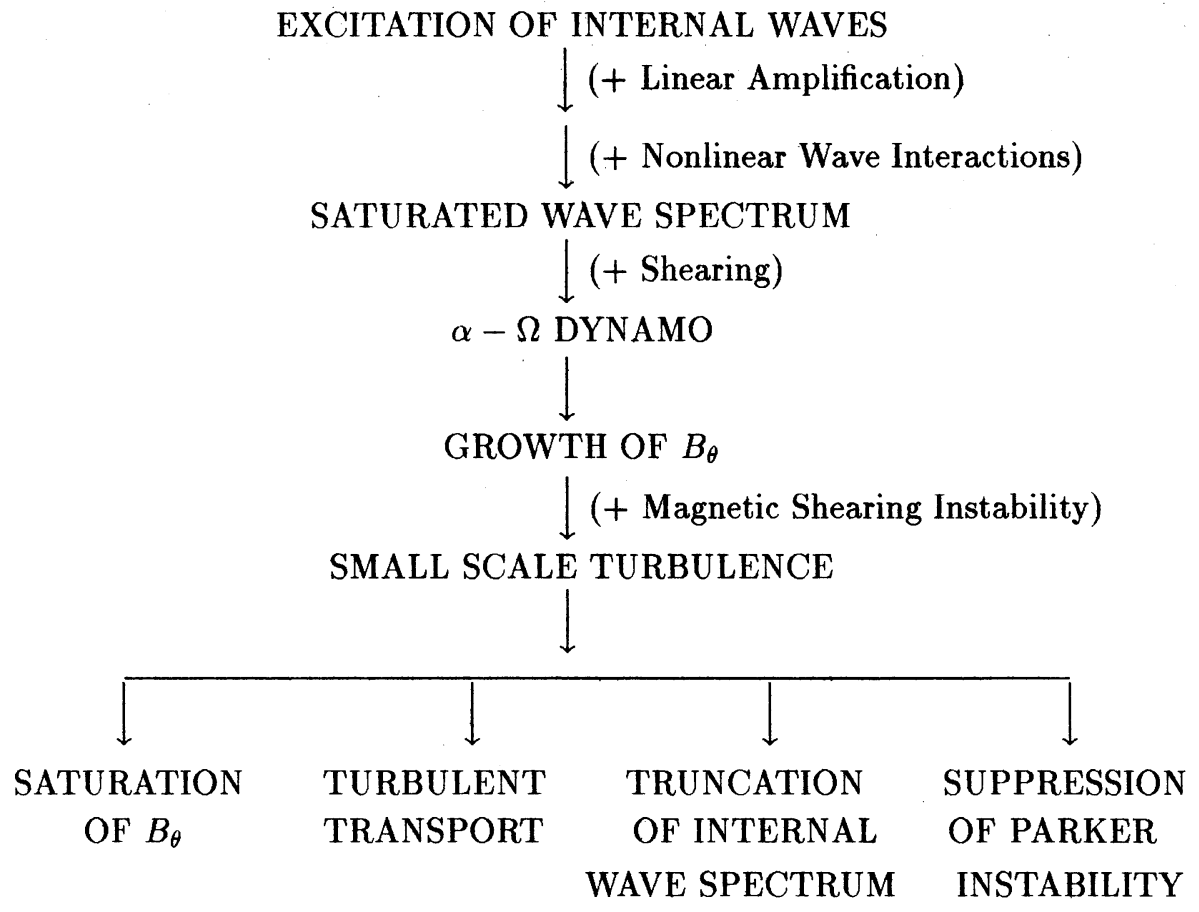

\section{Local Modes}

The process we have outlined above can be seen as the result of the interaction of various local modes. Here we briefly list the available modes and their role in our model. One critical point is that the physically important frequency is the one measured by an observer following local circular orbit. This is $\bar{\omega} \equiv \omega+m \Omega$ where $m$ 
is the azimuthal wavenumber. It is important to remember that this implies that a mode with a given frequency will have properties, determined by $\bar{\omega}$ that change over a typical distance of $r / m$. In listing the available modes we will assume the existence of a weak, azimuthal magnetic field. Such a field is generated self-consistently within this model.

The most interesting hydrodynamic modes are internal waves, analogous to deep ocean waves. For small $m$ they have a local dispersion relation of

$$
\bar{\omega}^{2}=\left(\frac{N^{2} k_{r}^{2}+\Omega^{2} k_{z}^{2}}{k^{2}}\right) .
$$

This implies that such waves are confined between a radius of reflection, where $\bar{\omega}^{2}=\Omega^{2}$ and a radius of resonant absorption where $\bar{\omega}^{2}=0$ and the wave energy piles up and undergoes nonlinear dissipation. (At a wave approaches this radius it is confined to an increasingly thin zone where $\bar{\omega}^{2}>N^{2}$.) These waves are strongly confined to the region where $N^{2}<\Omega$, i.e. close to the midplane of the disk, but over a broad enough region that they will affect the bulk of the disk gas. Moreover they are strongly dispersive, so their nonlinear interactions will tend to be incoherent and allow a higher wave amplitude. Finally, they are well-suited to driving a dynamo (Vishniac, Jin, and Diamond 1990), although there are some subtle points in driving a dynamo with waves. In view of these advantages it would appear to be a pity that they are typically confined to an annulus of width $r / m$. Fortunately this limitation does not apply to waves with $|m|=1$ (or $m=0$, but such waves turn out to be uninteresting for other reasons). Sound waves are also present, but are probably uninteresting for reasons given elsewhere.

A second set of interesting modes are Alfven waves polarized so that they correspond (more or less) to radial oscillations of the field lines. Below we will refer to this as the Magnetic Shearing Instability (MSI). These modes turn out to be unstable, with a growth rate $\Gamma \sim(m / r) V_{A}$ with an upper limit somewhat less than $\Omega$. This instability was first discovered by Velikhov (1959) and is described by Chandrasekhar (1961). Balbus and Hawley (1991) pointed out their significance for accretion disks. We have argued elsewhere (Vishniac and Diamond 1992) that these modes saturate with perturbation velocities comparable to $V_{A}$ and give rise to more or less isotropic MHD turbulence with a maximum scale of $\sim V_{A} / \Omega$. Still larger scale modes are suppressed by the smaller scale ones. The resulting turbulence tends to transport angular momentum outward with an effective viscosity of $V_{A}^{2} / \Omega$.

Finally, there is the Parker instability with $k_{z} \sim 1 / H$ and a growth rate of $V_{A} / H$. We mention these modes only because they have frequently been cited as the major factor preventing the growth magnetic fields in disks. However, these modes have a short radial wavelength, $\sim V_{A} / \Omega$, imposed by their slow growth rate and the rapid differential shearing within the disk. Consequently they are strongly disrupted by the MSI. They may survive at some level, but are unlikely to remove magnetic flux from the disk faster than turbulent diffusion. 


\section{The Internal Wave Driven Dynamo}

Why are $|m|=1$ internal waves so interesting? First, we note that if a wave is excited at some large radius with a low frequency then $\bar{\omega}=\Omega-\omega$ is never greater than $\Omega$, i.e. internal waves with this property will not encounter a reflecting surface as they propagate inward. The excitation of such waves will result from tidal effects (Goodman 1992), and may also come from the direct effect of the accretion stream itself. In both cases the waves will tend to have $\omega=\omega_{\text {binary }}<\Omega$.

The second point is that these waves tend to amplify as they propagate inward. Their energy flux is a combination of $\left\langle\delta P v_{r}\right\rangle$, roughly corresponding to the transport of some locally measurable energy density moving at the group velocity of the waves, and $\rho r \Omega\left\langle v_{r} v_{\theta}\right\rangle$, which is a contribution due to the energy caused by the angular momentum carried by the waves. As a wave goes by the mean orbital speed of the fluid temporarily decreases, corresponding to a drop in the orbital energy of the fluid. The latter contribution to the energy flux is equal to the angular momentum flux times $\Omega$. It can be shown that inward propagating internal waves with the desired properties are waves that have a positive angular momentum flux so the contribution to the energy flux from this term is also positive. Since the first term consists of a positive quantity (the local energy density being quadratic in the perturbation variables) being transported inward its contribution is negative. To linear order both the total energy flux and the angular momentum flux are conserved, implying that the second term grows proportionate to $\Omega$ and the first, being of opposite sign, must also grow. The result is that $\left\langle v^{2}\right\rangle$ increases as a wave propagates inward at a rate of roughly $m V_{\text {group }} / r \sim(H / r) c_{s}$. Since the waves are confined to the vicinity of the midplane, this linear growth can only be balanced by nonlinear dissipation. Internal waves are basically dispersive so we can approximate the nonlinear interaction rate as $\sim k^{2}\left\langle v^{2}\right\rangle / \bar{\omega}$. Balancing the linear growth and the nonlinear dissipation we conclude that $\left\langle v^{2}\right\rangle \sim(H / r) c_{s}^{2}$ for these waves. Of course, the presence of nonlinear dissipation implies that energy is being transferred to higher $m$ modes, a point we will return to shortly.

Finally, these waves have the capacity to drive an $\alpha-\Omega$ dynamo. This is not obvious and requires some explanation. Such a dynamo grows through a combination of shearing and some net helicity contributed by small scale motions. In order for growth to occur we need some finite $\alpha_{\theta \theta}$ to generate $B_{r}$ from $B_{\theta}$. Given this the field will grow at a rate of $\left(\alpha_{\theta \theta} \Omega / H\right)^{1 / 2}$ provided that dissipative effects are small. By definition $\alpha_{\theta \theta}=\left\langle v_{z} \partial_{\theta} v_{r}\right\rangle / r$. At any one moment internal waves can certainly contribute a nonzero $\alpha_{\theta \theta}$. The dominant contribution will come from waves with $k_{z} \sim 1 / H$, but it can be shown that such waves will tend to dominate the wave spectrum (Vishniac and Diamond, 1989). Of course, a truly periodic wave will give a contribution that averages to zero, but these waves are not completely periodic. They have a decorrelation rate of $\sim(H / r) \Omega$ due to nonlinear interactions. It is also true that if the wave spectrum is balanced, in the sense that waves are as likely to be coming from small $r$ as large, then summing over all wave modes gives zero, but the wave spectrum will heavily favor waves coming in from large $r$. Waves traveling the opposite direction will tend to diminish, rather than amplify, due to linear effects. Also, ingoing waves with suitably small frequencies are easy to generate. Outgoing 
waves would be expected to have large frequencies and travel only short distances before undergoing nonlinear dissipation. The end result is that the $|m|=1$ waves will result in $\alpha_{\theta \theta} \sim(H / r)^{3} c_{s}$, and a dynamo growth rate $\sim(H / r)^{3 / 2} \Omega$. Including contributions from small scale internal waves may increase this growth rate by a modest factor.

When the magnetic field has an infinitesimal strength then the only turbulent dissipation is caused by the waves themselves, which are unable to damp the dynamo. However, as the field strength increases the MSI takes over. The resulting turbulence disrupts the small scale internal waves (thereby lowering $\Gamma_{d y n a m o}$ and increasing the rate of turbulent flux loss from the disk). Saturation is defined by $\Gamma_{\text {dynamo }} \sim D / H^{2} \sim\left(V_{A} / c_{s}\right)^{2} \Omega$. Combining these considerations with a crude model for the internal wave spectrum (cf. Vishniac and Diamond, 1992) yields a saturated state with $V_{A} \sim(H / r)^{2 / 3} c_{s}$, and $\Gamma_{d y n a m o} \sim(H / r)^{4 / 3} \Omega$. The corresponding value of dimensionless 'viscosity' is $\sim(H / r)^{4 / 3}$ with a rather uncertain coefficient. We note that on scales of $\sim V_{A} / \Omega$ a small scale, rapidly varying, field is present which is as large (roughly) as the large scale field.

\section{Conclusions}

We have presented a model for a dynamo in accretion disks based on the action of internal waves propagating from the outer edge of the disk. As the magnetic field grows it gives rise to shearing instabilities which transport angular momentum outward and magnetic flux vertically. These instabilities will eventually saturate the dynamo when $\beta$ is still quite large. The resulting angular momentum transport is consistent with observations, although the prediction is rather approximate and is not strictly equivalent to a local viscosity in any case. From the point of view of dynamo theory the interesting feature here is a dynamo model which is nonturbulent, in the sense that the turbulent motions present are not responsible for driving the large scale magnetic field. ¿From the point of view of accretion disk theory the interest of this model is that it provides a way of understanding angular momentum transport in accretion disks without resort to phenomenological considerations.

\section{Acknowledgements}

This work is the result of a continuing collaboration with P.H. Diamond. Liping Jin and Min Huang have also made significant contributions. Support has been provided by NASA through contract NAGW 2048.

\section{References}

Balbus, S. and Hawley, J.: 1991, ApJ 376, 214

Chandrasekhar, S.: 1961, Hydrodynamic and Hydromagnetic Stability, Oxford University Press:London

Goodman, J.: 1992, ApJ ,

Shakura, N.I. and Sunyaev, R.A.: 1973, $A A$ 22, 471

Velikhov, E.P.: 1959, Soviet JETP 35, 1398

Vishniac, E.T. and Diamond, P.H.: 1989, ApJ 347, 435

Vishniac, E.T., Jin, L. and Diamond, P.H.: 1990, ApJ 365, 552

Vishniac, E.T. and Diamond, P.H.: 1992, ApJ 398, 561 